Алгебра и анализ

Том 22 (2010), № 5
St. Petersburg Math. J.

Vol. 22 (2011), No. 5, Pages 795-801

S 1061-0022(2011)01168-9

Article electronically published on June 28, 2011

\title{
EXTREMAL PROPERTIES OF SPHERICAL SEMIDESIGNS
}

\author{
N. O. KOTELINA AND A. B. PEVNYI
}

\begin{abstract}
For every even $t \geq 2$ and every set of vectors $\Phi=\left\{\varphi_{1}, \ldots, \varphi_{m}\right\}$ on the sphere $S^{n-1}$, the notion of the $t$-potential $P_{t}(\Phi)=\sum_{i, j=1}^{m}\left[\left\langle\varphi_{i}, \varphi_{j}\right\rangle\right]^{t}$ is introduced. It is proved that the minimum value of the $t$-potential is attained at the spherical semidesigns of order $t$ and only at them. The first result of this type was obtained by B. B. Venkov. The result is extended to the case of sets $\Phi$ that do not lie on the sphere $S^{n-1}$. For the V. A. Yudin potentials $U_{k}(\Phi), k=2,4, \ldots, t$, it is shown that they attain the minimal value at the spherical semidesigns of order $t$ and only at them.
\end{abstract}

\section{§1. Notation AND PRELIMINARY INFORMATION}

We use the dot product $\langle x, y\rangle=x_{1} y_{1}+\cdots+x_{n} y_{n}$ of vectors $x, y \in \mathbb{R}^{n}$ and the norm $\|x\|=\sqrt{\langle x, x\rangle}$.

Let

$$
S^{n-1}=\left\{x \in \mathbb{R}^{n}:\|x\|=1\right\}
$$

be the unit sphere in $\mathbb{R}^{n}$. In the sequel, we denote by $t$ an even number, $t \geq 2$.

Definition 1. A set of vectors $\Phi=\left\{\varphi_{1}, \varphi_{2}, \ldots, \varphi_{m}\right\} \subset S^{n-1}$ is called a spherical semidesign of order $t$ (or, simply, a spherical t-semidesign) if there is a constant $A_{t}>0$ such that the Waring identity

$$
\sum_{i=1}^{m}\left[\left\langle\varphi_{i}, x\right\rangle\right]^{t}=A_{t}\|x\|^{t}, \quad x \in \mathbb{R}^{n}
$$

is valid.

The constant $A_{t}$ can be found by successive applications of the Laplace operator $\Delta$ to both sides of equation (1). As a result, we obtain $A_{t}=\mathrm{cm}$, where

$$
c=\frac{(t-1) ! !}{n(n+2) \cdots(n+t-2)} .
$$

We also prove that a spherical semidesign of order $t$ is a spherical semidesign of all orders $k=2,4, \ldots, t$. The spherical $p$-designs were defined by Delsarte, Goethals, and Seidel in [4] in 1977. The same definition was used by many other authors [2, 4, 11, 12.

Definition 2. A set of points $\Phi=\left\{\varphi_{1}, \ldots, \varphi_{m}\right\} \subset S^{n-1}$ is called a spherical p-design if the identity

$$
\frac{1}{\sigma_{n}} \int_{S^{n-1}} Q(x) d S=\frac{1}{m} \sum_{i=1}^{m} Q\left(\varphi_{i}\right)
$$

is valid for all polynomials $Q(x)$ of degree at most $p$.

2010 Mathematics Subject Classification. Primary 52C35.

Key words and phrases. Spherical designs, spherical semidesigns.

(C)2011 American Mathematical Society 
Here $\sigma_{n}=2 \pi^{n / 2} / \Gamma\left(\frac{n}{2}\right)$ is the area of the sphere $S^{n-1}$, the order $p$ can be odd, and the polynomial $Q(x)$ is not necessarily homogeneous.

It is easy to establish a relationship between Definitions 1 and 2. If $\Phi=\left\{\varphi_{1}, \ldots, \varphi_{m}\right\}$ is a spherical semidesign of even order $t$, then

$$
\Phi_{2 m}=\left\{\varphi_{1}, \ldots, \varphi_{m},-\varphi_{1}, \ldots,-\varphi_{m}\right\}
$$

is a spherical design of order $t+1$.

Conversely, let the set $\Phi_{2 m} \subset S^{n-1}$ be symmetric, i.e., for each vector $\varphi \in \Phi_{2 m}$ we have $-\varphi \in \Phi_{2 m}$. Then $\Phi_{2 m}$ can be represented in the form

$$
\Phi_{2 m}=\Phi \cup-\Phi, \quad \Phi \cap-\Phi=\varnothing .
$$

If $\Phi_{2 m}$ is a spherical ( $\left.t+1\right)$-design, then $\Phi$ is a spherical semidesign of order $t$.

It should be noted that there exist nonsymmetric spherical designs. Interesting examples were presented in the papers [11, 12] by Yudin.

\section{§2. The inequality of B. B. Venkov}

We consider an arbitrary set $\Phi=\left\{\varphi_{1}, \ldots, \varphi_{m}\right\}$ of vectors on the sphere $S^{n-1}$. By the $t$-potential of this set we mean the quantity

$$
P_{t}(\Phi)=\sum_{i=1}^{m} \sum_{j=1}^{m}\left[\left\langle\varphi_{i}, \varphi_{j}\right\rangle\right]^{t}
$$

For $t=2$, the potential $P_{2}(\Phi)$ is called a frame. This potential was introduced in the papers [7, 8].

Theorem 1 (B. B. Venkov [1). For every set $\Phi=\left\{\varphi_{1}, \ldots, \varphi_{m}\right\} \subset S^{n-1}$, we have

$$
P_{t}(\Phi) \geq \mathrm{cm}^{2},
$$

where the constant $c$ is defined by (2). Equality occurs in (5) on the spherical $t$-semidesigns and only on them.

In 1, only spherical sets $\Phi$ were considered, and in this case, the minimum value of $P_{t}(\Phi)$ is attained at spherical $t$-designs and only at them. In the case of arbitrary sets $\Phi$ on the sphere, the minimal value of $P_{t}(\Phi)$ is attained at spherical semidesigns of order $t$. In the present paper, we generalize Theorem 1 to the case of nonunit vectors (see Theorem 2 below).

Example 1. Consider the set $\Phi=\left\{\varphi_{1}, \ldots, \varphi_{6}\right\}$ of six vertices of an icosahedron inscribed in $S^{2}$ such that $\Phi$ contains no opposite vectors. We calculate the 4-potential $P_{4}(\Phi)$.

Since $\varphi_{i} \in S^{2}$, we obtain $\left\|\varphi_{i}\right\|^{2}=1, i=1, \ldots, 6$. By Haantjes's theorem [6], the following relations are valid for the vertices of the icosahedron:

$$
\left\langle\varphi_{i}, \varphi_{j}\right\rangle= \pm \frac{1}{\sqrt{5}} \text { for } i \neq j
$$

Therefore, $P_{4}(\Phi)=6 \cdot 1+30 \cdot \frac{1}{25}=\frac{36}{5}$. At the same time, for $n=3, t=4$, and $m=6$, we have

$$
\mathrm{cm}^{2}=\frac{1 \cdot 3}{3 \cdot 5} \cdot 6^{2}=\frac{36}{5} .
$$

Since $P_{4}(\Phi)=\mathrm{cm}^{2}, \Phi$ is a 4 -semidesign. The set $\Phi_{12}=\left\{\varphi_{1}, \ldots, \varphi_{6},-\varphi_{1}, \ldots,-\varphi_{6}\right\}$ of all 12 vertices of the icosahedron is a spherical 5-design. 


\section{§3. NONSPHERICAL SEMIDESIGNS}

Now we consider $t$-semidesigns in $\mathbb{R}^{n}$ containing vectors with different lengths. Let $t$ be even, $t \geq 2$.

Definition 3. A set of nonzero vectors $\Phi=\left\{\varphi_{1}, \ldots, \varphi_{m}\right\}$ in $\mathbb{R}^{n}$ is called a $t$-semidesign if there is $A_{t}>0$ such that

$$
\sum_{i=1}^{m}\left[\left\langle\varphi_{i}, x\right\rangle\right]^{t}=A_{t}\|x\|^{t}, \quad x \in \mathbb{R}^{n} .
$$

Lemma 1. Let $t \geq 4$, and let $\Phi$ be a t-semidesign. Then the set of vectors

$$
\theta_{i}=\left\|\varphi_{i}\right\|^{2 /(t-2)} \varphi_{i}, \quad i=1, \ldots, m,
$$

is a $(t-2)$-semidesign with the constant

$$
A_{t-2}=A_{t} \frac{t+n-2}{t-1}
$$

Proof. Applying the Laplace operator $\Delta$ to both sides of equation (6), we obtain

$$
t(t-1) \sum_{i=1}^{m}\left[\left\langle\varphi_{i}, x\right\rangle\right]^{t-2}\left\|\varphi_{i}\right\|^{2}=A_{t} t(t+n-2)\|x\|^{t-2}, \quad x \in \mathbb{R}^{n} .
$$

This implies the claim.

We put $s=t / 2$. Applying the operator $\Delta s-1$ times, we arrive at the identity

$$
\sum_{i=1}^{m}\left[\left\langle\varphi_{i}, x\right\rangle\right]^{2}\left\|\varphi_{i}\right\|^{t-2}=A_{2}\|x\|^{2}, \quad x \in \mathbb{R}^{n}
$$

Applying the operator $\Delta$ yet another time, we obtain

$$
2 \sum_{i=1}^{m}\left\|\varphi_{i}\right\|^{t}=A_{2} \cdot 2 n
$$

Hence $A_{2}=\mu / n$, where

$$
\mu=\sum_{i=1}^{m}\left\|\varphi_{i}\right\|^{t}
$$

The recurrence relation in the lemma and the equation $A_{2}=\mu / n$ imply that $A_{t}=c \mu$, where $c$ is defined by (2).

\section{$\S 4$. An inequality for the $t$-Potential}

The $t$-potential $P_{t}(\Phi)$ is defined as above by formula (4).

Theorem 2. For every set $\Phi$ of vectors in the space $\mathbb{R}^{n}$, we have

$$
P_{t}(\Phi) \geq c\left(\sum_{i=1}^{m}\left\|\varphi_{i}\right\|^{t}\right)^{2}
$$

where the constant $c$ is defined by (2). Equality in (8) is attained at the t-semidesigns and only at them. 
For the proof, we need a certain technique involving a dot product in the space of homogeneous polynomials $\mathcal{P}_{n, t}$ of degree $t$.

For polynomials $f, g \in \mathcal{P}_{n, t}$,

$$
f(x)=\sum_{|i|=t} a(i) x^{i}, \quad g(x)=\sum_{|i|=t} b(i) x^{i},
$$

where $i=\left(i_{1}, \ldots, i_{n}\right)$ is a vector with nonnegative integral components (multi-index), $|i|=i_{1}+\cdots+i_{n}$, and $x^{i}=x_{1}^{i_{1}} x_{2}^{i_{2}} \ldots x_{n}^{i_{n}}$, we define the dot product

$$
[f, g]=\sum_{|i|=t} \frac{a(i) b(i)}{c(i)}, \quad \text { where } c(i)=\frac{t !}{i_{1} ! i_{2} ! \cdots i_{n} !} .
$$

Supplied with this dot product, $\mathcal{P}_{n, t}$ becomes a Hilbert space. For every $\varphi \in \mathbb{R}^{n}$, we consider the polynomial

$$
\rho_{\varphi}(x)=[\langle\varphi, x\rangle]^{t}=\left[\varphi_{1} x_{1}+\cdots+\varphi_{n} x_{n}\right]^{t} .
$$

It is easy to verify that for every $\varphi \in \mathbb{R}^{n}$ and $f \in \mathcal{P}_{n, t}$ we have

$$
\left[\rho_{\varphi}, f\right]=f(\varphi)
$$

i.e., $\rho_{\varphi}(x)$ is the reproducing kernel of the space $\mathcal{P}_{n, t}$. We also consider the polynomial $\omega_{t}(x)=\|x\|^{t}$ (as above, $t$ is an even number, $t \geq 2$ ). In [1] and [2], the following relation was proved:

$$
\left[\omega_{t}, \omega_{t}\right]=\frac{1}{c},
$$

where $c$ is a constant of the form (2).

Proof. The proof of Theorem 2 follows the idea indicated by B. B. Venkov in [1]. Using the notation $\mu$ introduced in (7), we can represent the required inequality in the form $P_{t}(\Phi) \geq c \mu^{2}$. By the inequality

$$
W:=\left[\sum_{i=1}^{m} \rho_{\varphi_{i}}-c \mu \omega_{t}, \sum_{j=1}^{m} \rho_{\varphi_{j}}-c \mu \omega_{t}\right] \geq 0,
$$

we obtain

$$
W=W_{1}-2 c \mu W_{2}+c^{2} \mu^{2} \cdot\left[\omega_{t}, \omega_{t}\right] .
$$

By (9), we have

$$
\begin{aligned}
W_{1} & =\sum_{i, j=1}^{m}\left[\rho_{\varphi_{i}}, \rho_{\varphi_{j}}\right]=\sum_{i, j=1}^{m} \rho_{\varphi_{j}}\left(\varphi_{i}\right)=\sum_{i, j=1}^{m}\left[\left\langle\varphi_{i}, \varphi_{j}\right\rangle\right]^{t}=P_{t}(\Phi), \\
W_{2} & =\sum_{i=1}^{m}\left[\rho_{\varphi_{i}}, \omega_{t}\right]=\sum_{i=1}^{m} \omega_{t}\left(\varphi_{i}\right)=\sum_{i=1}^{m}\left\|\varphi_{i}\right\|^{t}=\mu .
\end{aligned}
$$

Consequently, using (10), we see that

$$
W=P_{t}(\Phi)-2 c \mu^{2}+c^{2} \mu^{2} \frac{1}{c}=P_{t}(\Phi)-c \mu^{2} .
$$

Since $W \geq 0$, we have $P_{t}(\Phi) \geq c \mu^{2}$, which is equivalent to (8).

Now, we study when equality is attained.

Let the set $\Phi$ be a $t$-semidesign. In (6), we put $x=\varphi_{j}$ and use the fact that $A_{t}=c \mu$. We obtain

$$
\sum_{i=1}^{m}\left[\left\langle\varphi_{i}, \varphi_{j}\right\rangle\right]^{t}=c \mu\left\|\varphi_{j}\right\|^{t}, \quad j=1, \ldots, m .
$$


Performing summation in (11), we obtain $P_{t}(\Phi)=c \mu^{2}$.

Conversely, suppose equality in (8) occurs for some set $\Phi$. Then the quantity $W$ is equal to zero. The definition of $W$ shows that

$$
\sum_{i=1}^{m} \rho_{\varphi_{i}}(x)-c \mu \omega_{t}(x)=0, \quad x \in \mathbb{R}^{n}
$$

i.e., relation (6) is satisfied with the constant $A_{t}=c \mu$. Therefore, $\Phi$ is a $t$-semidesign. The theorem is proved.

\section{§5. Hehenbauer polynomials and a theorem of V. A. Yudin}

We fix a positive integer $n \geq 3$ and consider the weight

$$
w_{n}(u)=\left(1-u^{2}\right)^{(n-3) / 2}, \quad u \in[-1,1] .
$$

Let $G_{k}(u)$ be the Hehenbauer polynomials (ultraspherical polynomials) of degree $k$. They are orthogonal with weight $w_{n}(u)$,

$$
\int_{-1}^{1} G_{k}(u) G_{s}(u) w_{n}(u) d u=0, \quad k \neq s
$$

(see 9]). For $k=0$, we have $G_{0}(u) \equiv 1$. For $k \geq 1$, the polynomials $G_{k}(u)$ contain only even or only odd powers of $u$ depending on whether $k$ is even or odd. The addition formula

$$
G_{k}(\langle x, y\rangle)=\sum_{j=1}^{N(k)} Y_{j}(x) Y_{j}(y), \quad x, y \in S^{n-1}
$$

is valid, where the $Y_{j}, j=1, \ldots, N(k)$, are spherical functions of order $k$ (see [10]).

Let $\Phi^{*}=\left\{\varphi_{1}^{*}, \ldots, \varphi_{m}^{*}\right\} \subset S^{n-1}$ be a spherical $t$-semidesign, where $t$ is even, $t \geq 2$.

In [12, Yudin proved a criterion for the sphericity of designs in terms of Hehenbauer polynomials.

Since our definition of a spherical $t$-semidesign differs from that in [12, it is necessary to change the statement of the theorem.

Theorem 3 (V. A. Yudin). A set $\Phi^{*} \in S^{n-1}$ is a spherical t-semidesign if and only if

$$
\sum_{i=1}^{m} G_{k}\left(\left\langle\varphi_{i}^{*}, x\right\rangle\right)=0, \quad x \in S^{n-1}, \quad k=2,4, \ldots, t .
$$

\section{§6. Yudin Potentials}

For every set $\Phi=\left\{\varphi_{1}, \ldots, \varphi_{m}\right\}$ on the sphere $S^{n-1}$, we define the Yudin potential

$$
U_{k}(\Phi)=\sum_{i=1}^{m} \sum_{j=1}^{m} G_{k}\left(\left\langle\varphi_{i}, \varphi_{j}\right\rangle\right), \quad k=1,2, \ldots, t .
$$

The motivation for the term "potential" is the fact that the expressions $U_{k}(\Phi)$ are nonnegative. Indeed, by the addition formula (13), we have

$$
U_{k}(\Phi)=\sum_{s=1}^{N(k)} \sum_{i=1}^{m} Y_{s}\left(\varphi_{i}\right) \sum_{j=1}^{m} Y_{s}\left(\varphi_{j}\right)=\sum_{s=1}^{N(k)}\left[\sum_{i=1}^{m} Y_{s}\left(\varphi_{i}\right)\right]^{2} \geq 0 .
$$

Thus, for every set $\Phi \subset S^{n-1}$, the Yudin potentials $U_{k}(\Phi)$ are nonnegative for all $k=1,2, \ldots, t$. 
In the case where $n=3$, so that the Hehenbauer polynomials become Legendre polynomials, the following theorem was stated in [13. In the proof of the "if" part, it is convenient to use Venkov's theorem.

Theorem 4. Let $t$ be an even number with $t \geq 2$. A set $\Phi^{*}=\left\{\varphi_{1}^{*}, \ldots, \varphi_{m}^{*}\right\}$ on the sphere $S^{n-1}$ is a spherical t-semidesign if and only if

$$
U_{k}\left(\Phi^{*}\right)=0, \quad k=2,4, \ldots, t .
$$

Proof. The "only if" part. For a spherical $t$-semidesign $\Phi^{*}$, the Yudin theorem implies equations (14). We substitute $x=\varphi_{j}^{*}$ in these equations and sum over $j=1, \ldots, m$. We obtain

$$
\sum_{i=1}^{m} \sum_{j=1}^{m} G_{k}\left(\left\langle\varphi_{i}^{*}, \varphi_{j}^{*}\right\rangle\right)=0, \quad k=2,4, \ldots, t,
$$

which is equivalent to (15).

The "if" part. We use Theorem 1. Assume that relations (15) are valid. We calculate the usual $t$-potential

$$
P_{t}\left(\Phi^{*}\right)=\sum_{i, j=1}^{m}\left[\left\langle\varphi_{i}^{*}, \varphi_{j}^{*}\right\rangle\right]^{t}
$$

and verify that it is equal to $\mathrm{cm}^{2}$, where $c$ is defined by formula (2).

The polynomial $u^{t}$ can be expanded in even Hehenbauer polynomials as follows: $u^{t}=$ $\sum_{k=0,2, \ldots, t} d(k) G_{k}(u)$. We substitute $u=\left\langle\varphi_{i}^{*}, \varphi_{j}^{*}\right\rangle$ and sum over $i, j \in 1: m$. Then

$$
P_{t}\left(\Phi^{*}\right)=\sum_{k=0,2, \ldots, t} d(k) \sum_{i, j=1}^{m} G_{k}\left(\left\langle\varphi_{i}^{*}, \varphi_{j}^{*}\right\rangle\right) .
$$

By (16) and the identity $G_{0}(u) \equiv 1$, we arrive at the formula

$$
P_{t}\left(\Phi^{*}\right)=d(0) m^{2} \text {. }
$$

To calculate $d(0)$, we multiply $u^{t}$ by $G_{0}$, using the dot product ( , ) in the space $L_{2}(-1,1)$ with weight $w_{n}(u)$,

$$
d(0)=\frac{\left(u^{t}, G_{0}\right)}{\left(G_{0}, G_{0}\right)}=\frac{\int_{-1}^{1} u^{t} w_{n}(u) d u}{\int_{-1}^{1} w_{n}(u) d u}=: \frac{I(t)}{I(0)} .
$$

For even $t$, the integral $I(t)$ is calculated as follows:

$$
I(t)=2 \int_{0}^{1} u^{t} w_{n}(u) d u=\int_{0}^{1} x^{(t-1) / 2}(1-x)^{(n-3) / 2} d x=\frac{\Gamma\left(\frac{t+1}{2}\right) \Gamma\left(\frac{n-1}{2}\right)}{\Gamma\left(\frac{t+n}{2}\right)} .
$$

For $t=0$, we obtain the integral $I(0)$. As a result, we have

$$
d(0)=\frac{\Gamma\left(\frac{t+1}{2}\right) \Gamma\left(\frac{n}{2}\right)}{\sqrt{\pi} \Gamma\left(\frac{t+n}{2}\right)}=\frac{(t-1) ! !}{n(n+2) \cdots(n+t-2)}=c,
$$

where $c$ is as in (2). From (17), we conclude that $P_{t}\left(\Phi^{*}\right)=\mathrm{cm}^{2}$, and, by Venkov's theorem, $\Phi^{*}$ is a spherical $t$-semidesign. The theorem is proved.

Remark 1. For a symmetric spherical $t$-semidesign of the form

$$
\Phi_{2 m}^{*}=\left\{\varphi_{1}^{*}, \ldots, \varphi_{m}^{*},-\varphi_{1}^{*}, \ldots,-\varphi_{m}^{*}\right\},
$$

the "only if" part of the theorem can be refined, namely, we have

$$
U_{k}\left(\Phi_{2 m}^{*}\right)=0 \text { for all } k=1,2,3, \ldots, t .
$$




\section{REFERENCES}

[1] B. Venkov, Réseaux et designs sphériques, Réseaux Euclidiens, Designs Sphériques et Formes Modulaires, Monogr. Enseign. Math., vol. 37, Enseignement Math., Géneve, 2001, pp. 10-86. MR1878745 (2002m:11061)

[2] B. Reznick, Sums of even powers of real linear forms, Mem. Amer. Math. Soc. 96 (1992), no. 463, 155 pp. MR.1096187 (93h:11043)

[3] V. N. Malozemov and A. B. Pevny̌̆, Equiangular tight frames, Probl. Mat. Anal., No. 39, S.Peterburg. Univ., St. Petersburg, 2009, pp. 3-25; English transl., J. Math. Sci. (N. Y.) 157 (2009), no. 6, 789-815. MR2544028 (2010f:42063)

[4] P. Delsarte, J. M. Goethals, and J. J. Seidel, Spherical codes and designs, Geom. Dedicata 6 (1977), 363-388. MR0485471 (58:5302)

[5] E. Bannai, A. Munemasa, and B. Venkov, The nonexistence of certain tight spherical designs, Algebra i Analiz 16 (2004), no. 4, 1-23; English transl., St. Petersburg Math. J. 16 (2005), no. 4, 609-625. MR2090848(2005e:05022)

[6] J. Haantjes, Equilateral point-sets in elliptic two- and three-dimensional spaces, Nieuw. Arch. Wiskund (2) 22 (1948), 355-362. MR0023530(9:369c)

[7] J. J. Benedetto and M. Fickus, Finite normalized tight frames. Frame potentials, Adv. Comput. Math. 18 (2003), no. 2-4, 357-385. MR1968126 (2004c:42059)

[8] P. G. Casazza, Custom building finite frames, Wavelets, Frames and Operator Theory, Contemp. Math., vol. 345, Amer. Math. Soc., Providence, RI, 2004, pp. 61-86. MR2066822 (2005f:42078)

[9] G. Szegő, Orthogonal polynomials, 4th ed., Amer. Math. Soc. Colloq. Publ., vol. 23, Amer. Math. Soc., Providence, RI, 1975. MR0372517 (51:8724)

[10] S. L. Sobolev, Introduction to the theory of cubature formulae, Nauka, Moscow, 1974. (Russian) MR 0478560 (57:18037)

[11] V. A. Yudin, On variations of designs, Problemy Peredachi Informatsii 35 (1999), no. 4, 68-73; English transl., Probl. Inf. Transm. 35 (1999), no. 4, 346-350. MR1737747 (2000k:94059)

[12] _ Rotation of spherical designs, Problemy Peredachi Informatsii 36 (2000), no. 3, 39-45; English transl., Probl. Inf. Transm. 36 (2000), no. 3, 230-236. MR1793356 (2001f:05021)

[13] N. N. Andreev and V. A. Yudin, An extremal location of points on a sphere, Mat. Prosveshchenie Ser. 3 vyp. 1 (1997), 115-125. (Russian)

Department of Mathematics, Syktyvkar State University, Syktyvkar 167001, Russia

E-mail address: nad7175@yandex.ru

Department of Mathematics, Syktyvkar State University, Syktyvkar 167001, Russia

E-mail address: pevnyi@syktsu.ru

Received 4/AUG/2009

Translated by THE AUTHORS 